\title{
Mixed Matrix Membrane Tip Extraction Coupled with UPLC-MS/MS for the Monitoring of Nonsteroidal Anti-Inflammatory Drugs in Water Samples
}

\author{
Thipashini Ganesan ${ }^{1,2}$, Nurul Hazirah Mukhtar ${ }^{1,2}$, Hong Ngee Lim $^{3}$ and Hong Heng See $1,2, * \mathbb{D}$ \\ 1 Centre for Sustainable Nanomaterials, Ibnu Sina Institute for Scientific and Industrial Research, \\ Universiti Teknologi Malaysia, 81310 UTM Johor Bahru, Johor, Malaysia \\ 2 Department of Chemistry, Faculty of Science, Universiti Teknologi Malaysia, 81310 UTM Johor Bahru, \\ Johor, Malaysia \\ 3 Department of Chemistry, Faculty of Science, Universiti Putra Malaysia, 43400 Serdang, Selangor, Malaysia \\ * Correspondence: hhsee@utm.my
}

Received: 2 January 2020; Accepted: 10 March 2020; Published: 13 March 2020

\begin{abstract}
An ultra-performance liquid chromatography-tandem mass spectrometry (UPLC-MS/MS) method, in combination with a mixed matrix membrane microextraction method for the quantification of nonsteroidal anti-inflammatory drugs (NSAIDs) in environmental water samples, is reported. The extraction device was prepared by casting well-dispersed polymeric bonded octadecyl $\left(\mathrm{C}_{18}\right)$ particles in a cellulose triacetate matrix solution onto commercially available $200 \mu \mathrm{L}$ micropipette tips. The membrane formed contains $25 \%$ of the adsorbent loading amount and was firmly attached to the inner wall of the membrane tip. The dynamic extraction was performed by withdrawing and dispensing the sample solution through the tip device for effective analyte adsorption, followed by the analyte desorption process into $40 \mu \mathrm{L}$ of methanol and acetonitrile (1:1) prior to UPLC-MS/MS analysis. NSAIDs-namely diclofenac, ibuprofen, indoprofen, naproxen and sulindac-were chosen as targeted analytes. Several extraction parameters were comprehensively optimized, including sample $\mathrm{pH}$ value, ionic strength, dynamic extraction cycle, desorption solvent and desorption time. The optimized conditions demonstrated a linear range from 0.25 to $500 \mathrm{ng} \mathrm{L}^{-1}$, with correlation coefficients $\left(r^{2}\right)$ from 0.9988 to 0.9992 and detection limits ranging from 0.08 to $0.40 \mathrm{ng} \mathrm{L}^{-1}$. The recoveries of the spiked water samples were between $92 \%$ and $99 \%$ and exhibited excellent precision relative to standard deviations (RSDs $\leq 4.9 \%$ ), and enrichment factors (EFs) were at 201-249 for the developed approach.
\end{abstract}

Keywords: microextraction; mixed matrix membrane; sample preparation; NSAIDs; UPLC-MS/MS

\section{Introduction}

The sample preparation method is commonly employed for the isolation of potential interference in the sample and, at the same time, enriches the targeted analyte at the ultra-trace level to achieve the detection limits. Although classical extraction methods such as liquid-liquid extraction (LLE) and solid phase extraction (SPE) are particularly preferred, the drawbacks often stated are the high running cost, high consumption of organic solvents and multistep separation techniques. In recent years, a range of advanced miniaturized solid-liquid extraction methods-such as solid phase microextraction (SPME) [1-3], micro-solid phase extraction ( $\mu$-SPE) $[4,5]$, solid phase membrane tip extraction (SPMTE) [6,7], stir bar sorptive extraction (SBSE) [8], thin film microextraction (TFME) $[9,10]$ and microextraction by packed sorbent (MEPS) [11] — have been proposed to reduce time as well as errors, leading to low consumption of the hazardous solvent and low cost. 
One interesting method termed mixed matrix membrane microextraction [12-14], which is a simple adsorb/desorb extraction method consisting of the adsorbent being incorporated into the polymer matrix, was reported. The microextraction method was evaluated by dipping a small piece of $\mathrm{C}_{18}$-MMM $(7 \times 7 \mathrm{~mm})$ in a stirred $10 \mathrm{~mL}$ sample solution, followed by a desorption process into $100 \mu \mathrm{L}$ of methanol prior to instrumental analysis. The membrane showed a high affinity for NSAIDs in different spiked water samples, with enrichment factors (EFs) from 79 to 82 , relative recoveries ranging from $84 \%$ to $104 \%$, and RSDs between $0.2 \%$ and $8.6 \%(n=9)$. Moreover, single-layer graphene (SLG), multiwall carbon nanotubes (MWCNTs) and polymeric bonded octadecyl $\left(\mathrm{C}_{18}\right)$ particles are examples of adsorbents that have successfully immobilized within the extraction membrane device to monitor the ultra-trace level of priority organic pollutants and pharmaceuticals in environmental waters $[12,13]$. This approach was proven to be a simplified experimental setup with low costs. Other variations of extraction based on a mixed matrix membrane were also reported with satisfactory practicability [15-17].

Recently, the contamination of pharmaceuticals in the environment has become a critical issue in environmental chemistry. Nonsteroidal anti-inflammatory drugs (NSAIDs) are a group of pharmaceuticals drugs that have been widely used to treat pain, fever and anti-inflammation $[18,19]$. However, the contamination level of these drugs in the environmental ecosystem has now reached alarming stages because of their continuous release into the environment. Diclofenac, together with 17 alpha-ethinylestradiol (EE2) and 17 beta-estradiol (E2), was included in the watch list of substances to monitor in the field of water policy in Europe $[18,20]$. Miniaturized membrane-based microextraction techniques have been proposed for the extraction of NSAIDs and organic pollutants from various matrices. For instance, hollow-fiber liquid-phase microextraction (HF-LPME) [21], electromembrane extraction (EME) [22-25] and SPME [26] are proven to be beneficial in minimizing organic solvent usage. Nevertheless, analyzing the trace organic compound remains complex in complicated matrices and a wide range of chemical properties of the analytes, and they can be at concentrations as low as $\mathrm{pg} / \mathrm{mL}$ in range $[27,28]$.

Herein, we demonstrate a mixed matrix membrane microextraction approach coupled with ultra-performance liquid chromatography-tandem mass spectrometry (UPLC-MS/MS) for the quantification of NSAIDs in water samples. For the first time, a mixed matrix membrane immobilized with a $\mathrm{C}_{18}$ adsorbent was firmly cast within the inner wall of a commercial $200 \mu \mathrm{L}$ micropipette tip. In addition, the proposed extraction procedures were performed dynamically by introducing the sample into the membrane tip for effective adsorption, followed by desorbing the analytes into microliters of organic solvent prior to instrumental analysis. This concept is entirely new, relative to the previous planar sheet membrane design, and the extraction was performed in stagnant condition $[12,13]$. As model analytes, NSAIDs—namely indoprofen, sulindac, naproxen, diclofenac and ibuprofen-were used to compare with previous studies on the mixed matrix membrane extraction method performed by our research groups [12]. The potential of the developed extraction method extracting the target NSAIDs was investigated, optimized, validated and applied to real water samples.

\section{Materials and Methods}

Cellulose triacetate $(\mathrm{CTA})$, potassium dihydrogen phosphate $\left(\mathrm{KH}_{2} \mathrm{PO}_{4}\right)$, sodium chloride $(\mathrm{NaCl})$, sodium hydroxide $(\mathrm{NaOH})$, sulindac, indoprofen, naproxen, diclofenac and ibuprofen were obtained from Aldrich (Buchs, Switzerland). Organic solvents (HPLC grade) namely acetonitrile (MeCN), methanol (MeOH), 2-propanol (2-PrOH) and toluene-were purchased from Merck (Darmstardt, Germany). Deionized (DI) water was produced on a Direct-Q3 ultrapure water system (Merck Millipore, Darmstadt, Germany) and used throughout the experiment. Other reagents and chemicals were analytical grade and used without any further purification.

Stock solutions of NSAIDs (diclofenac, ibuprofen, indoprofen, naproxen and sulindac) at a concentration of $100 \mu \mathrm{g} \mathrm{mL} \mathrm{m}^{-1}$ were dissolved using C-grade methanol and kept in a refrigerator. Standard solutions at concentrations of $100 \mathrm{ng} \mathrm{L}^{-1}$ were prepared daily by diluting the stock solutions 
with DI water. In this work, the water samples were taken from the outlet of an effluent treatment plant of the Universiti Teknologi Malaysia (UTM) Health Center in Skudai Johor, Malaysia. A $0.2 \mu \mathrm{m}$ polytetrafluoroethylene (PFTE) membrane filter (Macherey-Nagel $\mathrm{GmbH}$, Germany) was used to vacuum-filter the samples. For further use, the samples were stored at $4{ }^{\circ} \mathrm{C}$ in amber glass bottles capped with Teflon-lined caps for no longer than a month. A blank analysis of wastewater performed by conventional SPE polymeric bonded octadecyl $\left(\mathrm{C}_{18}\right)$ cartridges [12] using UPLC-MS/MS showed that the NSAIDs were free of contamination. Thus, the collected water samples were used to evaluate the analytical performance of the extraction method. The standard and spiked sample solutions were adjusted to $\mathrm{pH} 3.5$ using $1 \mathrm{M} \mathrm{HCl}$ prior to extraction.

Asymmetrical $C_{18}$ particles with an average size of $50 \mu \mathrm{m}$ and a surface area of $480 \mathrm{~m}^{2} \mathrm{~g}^{-1}$ were obtained from Macherey-Nagel (Düren, Germany). To prepare the mixed matrix membrane casting solution, $10 \mathrm{mg}$ of the $\mathrm{C}_{18}$ particles and $40 \mathrm{mg}$ of CTA were dissolved in $1 \mathrm{~mL}$ of dichloromethane (DCM) (equal to $25 \% \mathrm{w} / \mathrm{w}$ loading of $\mathrm{C}_{18}$ ). The casting solution was ultrasonicated for $5 \mathrm{~min}$ to achieve uniform dispersion. Next, $100 \mu \mathrm{L}$ of the casting solution was withdrawn into a $200 \mu \mathrm{L}$ pipette tip (Eppendorf, Hamburg, Germany) using a digital micropipette (Eppendorf, Hamburg, Germany). The end of the tip was covered firmly with Parafilm (Bemis, Neenah, WI, USA) to avoid the casting solution from spilling. The pipette tip was subsequently removed from the micropipette and placed in the pipette tip holder, and the solution was then solidified by evaporation in an oven with a temperature maintained at $35^{\circ} \mathrm{C}$ for approximately $30 \mathrm{~min}$. It was then removed from the pipette tip, and the membrane thickness was determined using digital micrometers (MDC-1, Mitutoyo Corporation, Kawasaki, Japan). The average thickness was approximately $50 \mu \mathrm{m}$.

The membrane tip was employed as the extraction device (Figure 1). The upper part of the fabricated membrane tip ( $\sim 7 \mathrm{~mm}$ in length) was cut off and firmly attached to a $10 \mathrm{~mL}$ disposable plastic syringe (Terumo, Leuven, Belgium). The membrane tip was immersed in methanol for conditioning for about $30 \mathrm{~min}$ to activate the sorbent surface, followed by rinsing with DI water. The sequence of dynamic extraction begins by withdrawing the sample solution into the syringe device, followed by dispensing the desorption solvent to collect the desorption extract (Figure 2). The procedure starts with introducing $10 \mathrm{~mL}$ of the sample solution through the membrane tip device by pulling the syringe plunger consistently at a constant flow rate of approximately $300 \mu \mathrm{L} \mathrm{s}^{-1}$ for an effective analyte adsorption process on the sample/membrane interface. The withdrawn sample was dispensed from the syringe, with a residence time of about $3 \mathrm{~s}$, through the membrane tip back into the sample vial for the second adsorption process at the same sample flowing speed. These dynamic extraction procedures were repeated ten times to achieve optimum adsorption efficiency, followed by the washing out the membrane tip wall lightly with DI water twice to remove any possible surface contamination. Following the adsorption and washing process, the membrane tip was removed from the syringe and reattached to a $1 \mathrm{~mL}$ plastic syringe (Terumo, Leuven, Belgium). The back extraction of NSAIDs from the membrane tip was performed by flushing the membrane tip wall with $40 \mu \mathrm{L}$ of methanol and acetonitrile (1:1) as a desorption solvent repetitively for ten cycles. Finally, an enriched extract of $5.0 \mu \mathrm{L}$ was injected into the UPLC-MS/MS system for separation and detection.

The UPLC-ESI-MS/MS system consists of a Waters Acquity UPLC H-class system and a Xevo TQ-S micro ESI mass spectrometer (Milford, MA, USA) controlled using MassLynx software (V4.1, Waters Inc., Milford, MA, USA). The chromatographic separations were carried out on the Waters UPLC BEH C ${ }_{18} 1.7 \mu \mathrm{m}(2.1 \mathrm{~mm} \times 100 \mathrm{~mm})$ column. The chromatographic parameters were maintained at a column temperature of $40^{\circ} \mathrm{C}$, with a flow rate of $0.3 \mathrm{~mL} \mathrm{~min}^{-1}$ and an injection volume of $5 \mu \mathrm{L}$. The binary mobile phase consists of $10 \mathrm{mM}$ ammonium acetate (A) at $100 \% \mathrm{MeOH}(\mathrm{B})$, and the NSAIDs were separated using a gradient elution method consisting of $40 \% \mathrm{~B}$ at $0 \mathrm{~min}, 50 \% \mathrm{~B}$ at $2 \mathrm{~min}$ and $70 \%$ $\mathrm{B}$ at $5 \mathrm{~min}$. A multiple reaction monitoring (MRM) method was established via syringe infusion of five NSAIDs to determine the MRM transitions. The MS parameters were as follows: MRM modes (ESI +/-), capillary voltage, $1.0 /-3.0 \mathrm{kV}$; cone voltage, $20 \mathrm{~V}$; source temperature, $150{ }^{\circ} \mathrm{C}$; desolvation 
temperature, $450{ }^{\circ} \mathrm{C}$; and desolvation gas flow rate, $800 \mathrm{~L} \mathrm{~h}^{-1}$, respectively. Finalised MRM transitions employed are illustrated in Table 1.

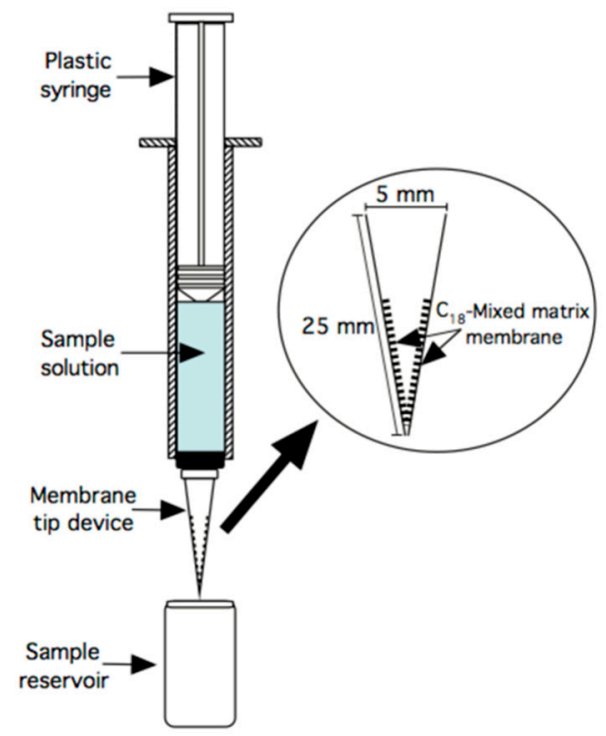

Figure 1. Schematic illustration of the $\mathrm{C}_{18}$-mixed matrix membrane tip microextraction setup.

(a)

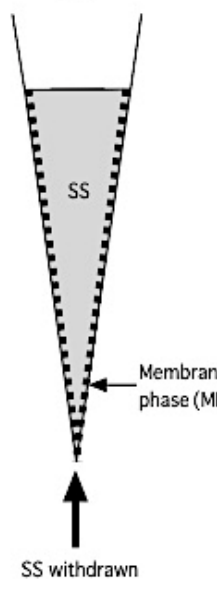

(b)

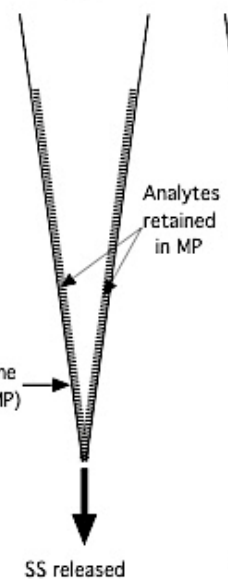

(c)

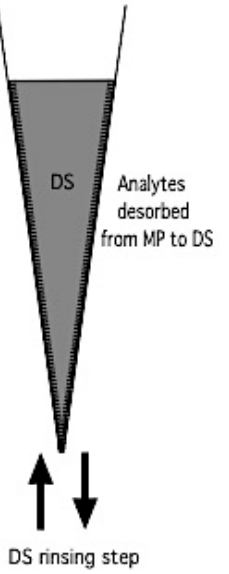

Figure 2. Expanded view of dynamic microextraction. (a) The sample solution (SS) is continuously withdrawn into the membrane tip for analyte adsorption. (b) The withdrawn SS is dispensed from the membrane tip into the sample vial, with the analytes retained in the membrane phase (MP). (c) The desorption solvent (DS) is continuously flushed in the membrane tip ten times, and the analytes are transferred rapidly from the MP to the DS.

Table 1. Finalized multiple reaction monitoring (MRM) transition by ultra-performance liquid chromatography-tandem mass spectrometry (UPLC-MS/MS) electrospray ionization polarity switch.

\begin{tabular}{cccccc}
\hline Analyte & $\begin{array}{c}\text { Retention } \\
\text { Time (min) }\end{array}$ & Polarity & Precursor Ion & Product Ion 1 & Product Ion 2 \\
\hline Indoprofen & 1.49 & Positive & 282.2 & 77 & 236.1 \\
Sulindac & 1.62 & Positive & 357.1 & 233.1 & 341.1 \\
Naproxen & 1.74 & Positive & 231.1 & 170 & 185.1 \\
Diclofenac & 2.40 & Positive & 296.1 & 215 & 250 \\
Ibuprofen & 2.55 & Negative & 205 & 161.1 & 172.9 \\
\hline
\end{tabular}




\section{Results and Discussion}

\subsection{Extraction Device Characterization}

The membrane composition employed in this work was directly adopted from our earlier research [12], and the extraction tip device preparation protocol was stated in Section 2. Representative surfaces and cross-sectional scanning electron microscope (SEM) images for the membrane tip device were obtained using a Hitachi SU-8020 field-emission SEM (Hitachi-Hitech, Tokyo, Japan) (Figure 3). Overall, the membrane was attached firmly, surrounding the pipette tip wall with a measured inner upper- and lower-end diameter of approximately 3 and $1 \mathrm{~mm}$, respectively (Figure 3a). The membrane's mechanical structure was investigated by detaching the membrane from the tip wall prior to characterization. As illustrated in Figure $3 b$, the membrane filled with $C_{18}$ particles has a dense structure. The cross-sectional image also clearly displays the tight and uniform distribution of $C_{18}$ particles. Therefore, these favorable structures are highly suitable for the proposed microextraction procedure. Furthermore, the dense structure observed on the upper surface also indicates that the high sponginess of a pure CTA polymer matrix has disappeared [29] and is substituted by $\mathrm{C}_{18}$ particles (Figure 3c). The inner membrane surface demonstrated an even and nonporous structure, and all the components were well combined to form a homogeneous membrane surface (Figure 3d).

(a)

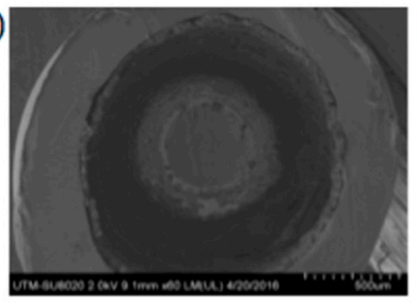

(c)

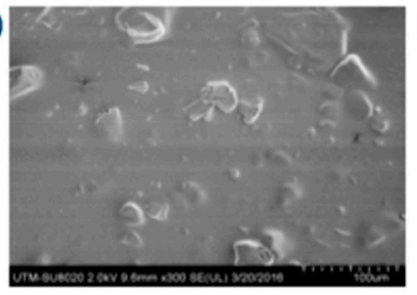

(b)

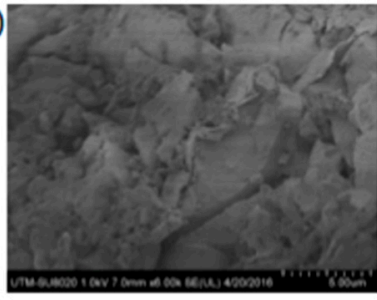

(d)

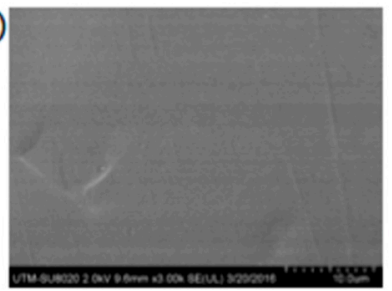

Figure 3. Field-emission SEM images of the $\mathrm{C}_{18}$-mixed matrix membrane tip device: (a) overall tip structure (top view); (b) cross-section image of membrane; (c) outer surface image of membrane; (d) inner surface image of membrane.

\subsection{Optimization of Extraction Conditions}

The operational parameters were elucidated with respect to the $\mathrm{pH}$ of the sample, ionic strength, dynamic extraction cycle, desorption solvent and desorption time to comprehensively study the performance of the protocol for mixed matrix membrane microextraction.

As the targeted NSAID analytes are adsorbed by the membrane, mainly via hydrophobic interaction, the $\mathrm{pH}$ of the sample solution should be maintained in the acidic medium to keep it in the molecular state. The sample $\mathrm{pH}$ values of $2.5,3.5,4,5$ and 6 were studied by adjusting with $1 \mathrm{M}$ $\mathrm{HCl}$ (Figure S1). Peak areas gradually increased from 2.5 and reached their highest when a pH of 3.5 was adopted. Further increases in the sample $\mathrm{pH}$ to 4,5 and 6 resulted in decreased peak areas as the targeted analytes started to be deprotonated into their ionic forms at higher $\mathrm{pH}$ values [30-32]. A similar trend was observed in the previous work [12]. Hence, a sample pH of 3.5 was chosen for the subsequent analyses.

Ionic strength was investigated as the addition of salt can decrease the solubility of analytes and, therefore, enhance the salting-out effects [7]. Hence, $\mathrm{NaCl}$ was added to the final concentrations of $0 \%$, $1 \%, 2 \%, 2.5 \%, 5 \%, 7.5 \%$ and $10 \%(\mathrm{w} / \mathrm{v})$ (Figure S2). The peak areas were highest when $2 \%(\mathrm{w} / \mathrm{v})$ of 
$\mathrm{NaCl}$ was added to the sample. However, adding more $\mathrm{NaCl}$ led to no improvement of extraction performance, possibly because of the increased viscosity of the sample [33]. Therefore, the following experiments were performed with the addition of $2 \%(\mathrm{w} / \mathrm{v}) \mathrm{NaCl}$ into the sample solution.

The extraction performance in this proposed new approach can be significantly improved by increasing the number of dynamic extraction cycles employed, which will directly increase the interaction of NSAID analytes in the sample solution and membrane surface. However, the use of excessively high numbers of extraction cycles will significantly prolong the total analysis time. Hence, extraction cycles in the range of two, four, six, eight, ten, twelve, fourteen and sixteen cycles were further optimized (Figure 4a). The peak areas were at their highest when ten extraction cycles were performed, which took about $10 \mathrm{~min}$, and reached a plateau when the cycles were increased to twelve, fourteen and sixteen. Thus, ten extraction cycles were performed for subsequent analyses.
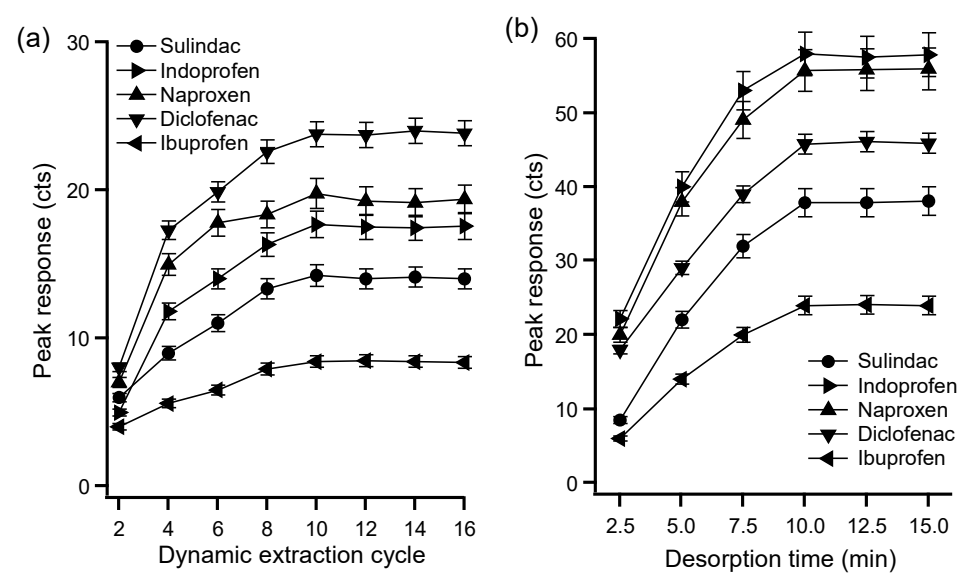

Figure 4. Effect of extraction cycle (a) and desorption time (b) on peak responses using mixed matrix membrane tip microextraction. Extraction parameters: sample $\mathrm{pH}, 3.5$; addition of salt, $2 \%(\mathrm{w} / \mathrm{v})$; sample volume, $10 \mathrm{~mL}$; desorption solvent volume, $40 \mu \mathrm{L}$; analyte concentration, $100 \mathrm{ng} \mathrm{L}^{-1}$ of spiked solution. (Error bars represent standard deviations of results, $n=3$ ).

Organic solvents compatible to the mobile phase of LC-MS-namely methanol, ethanol, 2-propanol, acetonitrile and a mixture of methanol and acetonitrile (1:1)-were evaluated as desorption solvents to retrieve the entrapped analytes by the desorption process. The use of methanol and acetonitrile (1:1) as desorption solvents generally exhibited the highest peak areas [30] among all the solvents being investigated.

The desorption step was then further investigated by examining the desorption rinsing time of 2.5, 5, 7.5, 10, 12.5 and $15 \mathrm{~min}$ (Figure $4 \mathrm{~b}$ ). The peak areas gradually increased with the increasing desorption time from $2.5 \mathrm{~min}$ onward, reached their highest at $10 \mathrm{~min}$ and reached a plateau when the desorption rinsing time was extended to 12.5 and $15 \mathrm{~min}$. Note that the volume of the desorption solvent was set at $40 \mu \mathrm{L}$, as this is the lowest volume that can be used to rinse the entire membrane wall using a $1 \mathrm{~mL}$ plastic syringe.

\subsection{Evaluation of Analytical Performance}

The optimum extraction conditions for the $10 \mathrm{~mL}$ sample solutions at a concentration of $10 \mathrm{ng} \mathrm{L}^{-1}$ were selected as follows: $\mathrm{pH} 3.5,2.0 \%$ of salt addition, a $40 \mu \mathrm{L}$ mixture of methanol and acetonitrile (1:1) as the desorption solvent, and a desorption time of $10 \mathrm{~min}$ under ultrasonication. The analytical figures of merit are summarized in Table 2. The calibration curves were plotted by the peak response of the analytes versus the concentration, and each concentration was performed in triplicate. The calibration plots of each analyte-prepared at $0.25-500 \mathrm{ng} \mathrm{L}^{-1}$ for indoprofen and diclofenac, $0.30-500 \mathrm{ng} \mathrm{L}^{-1}$ for sulindac, $0.50-500 \mathrm{ng} \mathrm{L}^{-1}$ for naproxen and $1.30-500 \mathrm{ng} \mathrm{L}^{-1}$ for ibuprofen-were observed to be linear for spiked sewage water samples, with correlation coefficients $\left(r^{2}\right)$ ranging between 0.9988 and 
0.9992. The overall detection limits (LODs) and quantification limits (LOQs) were $0.08-0.40 \mathrm{ng} \mathrm{L}^{-1}$ and $0.25-1.30 \mathrm{ng} \mathrm{L}^{-1}$, respectively, with EFs of 201-249. The repeatability of the spiked concentration of $10 \mathrm{pg} / \mathrm{mL}$ was satisfactory, with an intraday $(n=5)$ RSD of $4.3-4.9 \%$. This newly proposed approach was compared with the previously reported method, as listed in Table S1, and was proven able to provide superior detection sensitivity for trace-level analysis.

Table 2. Linear range, correlation coefficient $\left(r^{2}\right)$, detection limits (LODs), quantification limits (LOQs), repeatability, and enrichment factors (EFs) of nonsteroidal anti-inflammatory drugs (NSAIDs) in spiked water samples in combination with UPLC-MS/MS.

\begin{tabular}{|c|c|c|c|c|c|c|}
\hline & $\begin{array}{c}\text { Linearity Range } \\
\left(n g \mathrm{~L}^{-1}\right)\end{array}$ & $r^{2}$ & $\begin{array}{l}\operatorname{LOD}^{1} \\
\left(\mathrm{ng} \mathrm{L}^{-1}\right)\end{array}$ & $\begin{array}{c}\mathrm{LOQ}^{2} \\
\left(\mathrm{ng} \mathrm{L}^{-1}\right)\end{array}$ & $\begin{array}{l}\text { Repeatability }^{3} \\
\operatorname{RSD}(\%, n=5)\end{array}$ & $\mathrm{EF}^{4}$ \\
\hline Indoprofen & $0.25-500$ & 0.9992 & 0.08 & 0.25 & 4.3 & 202 \\
\hline Sulindac & $0.30-500$ & 0.9988 & 0.10 & 0.30 & 4.6 & 208 \\
\hline Naproxen & $0.50-500$ & 0.9990 & 0.15 & 0.50 & 4.5 & 201 \\
\hline Diclofenac & $0.25-500$ & 0.9989 & 0.08 & 0.25 & 4.3 & 241 \\
\hline Ibuprofen & $1.30-500$ & 0.9990 & 0.40 & 1.30 & 4.9 & 249 \\
\hline
\end{tabular}

Calculated from $\mathrm{S} / \mathrm{N}=3^{1}$ and $\mathrm{S} / \mathrm{N}=10^{2}$; From peak areas at $2 \mathrm{ng} \mathrm{L}^{-1}(n=5)$ of each NSAID using the same tip ${ }^{3}$; $\mathrm{EF}=$ the final concentration of analyte in the enriched extract/initial concentration of analyte in the sample solution ${ }^{4}$.

Fifteen batches of spiked sewage water samples from the same source, containing $10 \mathrm{ng} \mathrm{L}^{-1}$ of NSAIDs, were evaluated consecutively on the stability of the developed membrane tip microextraction device under optimum conditions. The results of RSDs $\leq 5 \%(n=10)$ proved that the membrane tip has achieved good stability. However, the peak areas then gradually decreased, and a loss of extraction efficiency of approximately $2-7 \%$ was shown for the eleventh and twelfth extraction compared to the first extraction. Membrane tip-to-tip variation was also investigated with RSDs $\leq 8 \%(n=8)$.

The water samples were spiked with NSAIDs at three concentration levels of 10, 100 and $500 \mathrm{ng} \mathrm{L}^{-1}$ under optimized conditions to evaluate the applicability and consistency of the developed method in real water samples The recoveries were between $92 \%$ and $99 \%(n=5)$ and with relative standard deviations of $4.3-4.9 \%$, proving that the extraction efficiencies were reliable and consistent as they were unaffected by the background of the water matrices. Figure 5 showed the UPLC-MS/MS chromatogram of NSAIDs separation when spiked in water sample after treatment with mixed matrix membrane tip extraction under optimum parameters. The targeted analytes achieved upconcentration after extraction.

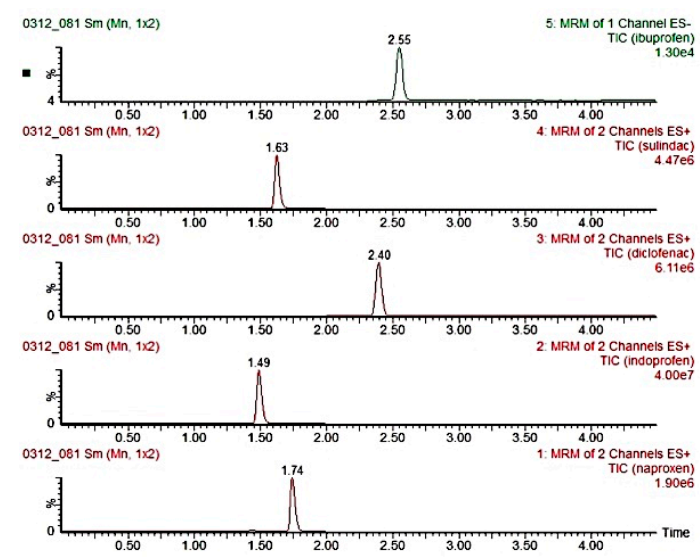

Figure 5. UPLC-MS/MS chromatograms showing the detection of five targeted NSAIDs extracted from effluent water samples spiked at $10 \mathrm{ng} \mathrm{L}^{-1}$.

\section{Conclusions}

A variation of mixed matrix membrane microextraction, based on a membrane tip platform, was successfully demonstrated. The fabrication steps of the membrane tip are simple and possible at 
a low cost because no special equipment is required. Unlike conventional techniques that require high amounts of organic solvents, the developed approach requires less consumption of the organic solvent (in the microliter range). In addition, the selectivity of the membrane tip can be easily manipulated using other adsorbent particles with unique functionality. Future investigations will focus on directly coupling the proposed approach with a mass spectrometry detector for a rapid and immediate "sample-in, answer-out" analytical platform.

Supplementary Materials: The following are available online at http://www.mdpi.com/2297-8739/7/1/19/s1, Figure S1: Effect of sample $\mathrm{pH}$ on peak responses using mixed matrix membrane tip microextraction, Figure S2: Effect of addition of salt on peak responses using mixed matrix membrane tip microextraction, Table S1: Comparison of the current work with other previous methods for analysis of NSAIDs.

Author Contributions: Conceptualization, T.G., N.H.M., H.N.L. and H.H.S.; methodology, T.G. and N.H.M.; writing — original draft preparation, T.G. and N.H.M.; writing—review and editing, T.G., N.H.M., H.N.L. and H.H.S. All authors have read and agreed to the published version of the manuscript.

Funding: This research was funded by Universit Teknologi Malaysia through UTM IIIG grant (Q.J130000.3054.01M38) and UTM Shine Signature Grant (Q.J130000.2454.07G73).

Conflicts of Interest: The authors declare no conflict of interest. The funders had no role in the design of the study; in the collection, analyses, or interpretation of data; in the writing of the manuscript, or in the decision to publish the results.

\section{References}

1. Souza-Silva, É.A.; Reyes-Garcés, N.; Gómez-Ríos, G.A.; Boyac1, E.; Bojko, B.; Pawliszyn, J. A critical review of the state of the art of solid-phase microextraction of complex matrices iii. Bioanalytical and clinical applications. TrAC-Trend Anal. Chem. 2015, 71, 249-264. [CrossRef]

2. Boyac1, E.; Rodríguez-Lafuente, Á.; Gorynski, K.; Mirnaghi, F.; Souza-Silva, É.A.; Hein, D.; Pawliszyn, J. Sample preparation with solid phase microextraction and exhaustive extraction approaches: Comparison for challenging cases. Anal. Chim. Acta 2015, 873, 14-30. [CrossRef] [PubMed]

3. Kataoka, H.; Ishizaki, A.; Saito, K. Recent progress in solid-phase microextraction and its pharmaceutical and biomedical applications. Anal. Methods 2016, 8, 5773-5788. [CrossRef]

4. Basheer, C.; Alnedhary, A.A.; Rao, B.S.M.; Valliyaveettil, S.; Lee, H.K. Development and application of porous membrane-protected carbon nanotube micro-solid-phase extraction combined with gas chromatography/mass spectrometry. Anal. Chem. 2006, 78, 2853-2858. [CrossRef]

5. Naing, N.N.; Li, S.F.Y.; Lee, H.K. Micro-solid phase extraction followed by thermal extraction coupled with gas chromatography-mass selective detector for the determination of polybrominated diphenyl ethers in water. J. Chromatogr. A 2016, 1458, 25-34. [CrossRef]

6. See, H.H.; Marsin Sanagi, M.; Ibrahim, W.A.W.; Naim, A.A. Determination of triazine herbicides using membrane-protected carbon nanotubes solid phase membrane tip extraction prior to micro-liquid chromatography. J. Chromatogr. A 2010, 1217, 1767-1772. [CrossRef]

7. Kamaruzaman, S.; Sanagi, M.M.; Endud, S.; Wan Ibrahim, W.A.; Yahaya, N. Mcm-41 solid phase membrane tip extraction combined with liquid chromatography for the determination of non-steroidal anti-inflammatory drugs in human urine. J. Chromatogr. B 2013, 940, 59-65. [CrossRef]

8. David, F.; Ochiai, N.; Sandra, P. Two decades of stir bar sorptive extraction: A retrospective and future outlook. TrAC-Trend Anal. Chem. 2019, 112, 102-111. [CrossRef]

9. Bruheim, I.; Liu, X.; Pawliszyn, J. Thin-film microextraction. Anal. Chem. 2003, 75, 1002-1010. [CrossRef]

10. Grandy, J.J.; Boyac1, E.; Pawliszyn, J. Development of a carbon mesh supported thin film microextraction membrane as a means to lower the detection limits of benchtop and portable gc/ms instrumentation. Anal. Chem. 2016, 88, 1760-1767. [CrossRef]

11. Moein, M.M.; Abdel-Rehim, A.; Abdel-Rehim, M. Microextraction by packed sorbent (meps). TrAC-Trend Anal. Chem. 2015, 67, 34-44. [CrossRef]

12. Kamaruzaman, S.; Hauser, P.C.; Sanagi, M.M.; Ibrahim, W.A.W.; Endud, S.; See, H.H. A simple microextraction and preconcentration approach based on a mixed matrix membrane. Anal. Chim. Acta 2013, 783, 24-30. [CrossRef] [PubMed] 
13. Mukhtar, N.H.; See, H.H. Carbonaceous nanomaterials immobilised mixed matrix membrane microextraction for the determination of polycyclic aromatic hydrocarbons in sewage pond water samples. Anal. Chim. Acta 2016, 931, 57-63. [CrossRef] [PubMed]

14. Mukhtar, N.H.; Mamat, N.A.; See, H.H. Monitoring of tobramycin in human plasma via mixed matrix membrane extraction prior to capillary electrophoresis with contactless conductivity detection. J. Pharmaceut. Biomed. Anal. 2018, 158, 184-188. [CrossRef]

15. Riasat Harami, H.; Riazi Fini, F.; Rezakazemi, M.; Shirazian, S. Sorption in mixed matrix membranes: Experimental and molecular dynamic simulation and grand canonical monte carlo method. J. Mol. Liq. 2019, 282, 566-576. [CrossRef]

16. Ghani, M.; Ghoreishi, S.M.; Azamati, M. Magnesium-aluminum-layered double hydroxide-graphene oxide composite mixed-matrix membrane for the thin-film microextraction of diclofenac in biological fluids. J. Chromatogr. A 2018, 1575, 11-17. [CrossRef]

17. Rozaini, M.N.H.; Semail, N.-f.; Saad, B.; Kamaruzaman, S.; Abdullah, W.N.; Rahim, N.A.; Miskam, M.; Loh, S.H.; Yahaya, N. Molecularly imprinted silica gel incorporated with agarose polymer matrix as mixed matrix membrane for separation and preconcentration of sulfonamide antibiotics in water samples. Talanta 2019, 199, 522-531. [CrossRef]

18. Barbosa, M.O.; Moreira, N.F.F.; Ribeiro, A.R.; Pereira, M.F.R.; Silva, A.M.T. Occurrence and removal of organic micropollutants: An overview of the watch list of eu decision 2015/495. Water Res. 2016, 94, 257-279. [CrossRef] [PubMed]

19. Ji, Y.; Du, Z.X.; Zhang, H.J.; Zhang, Y. Rapid analysis of non-steroidal anti-inflammatory drugs in tap water and drinks by ionic liquid dispersive liquid-liquid microextraction coupled to ultra-high performance supercritical fluid chromatography. Anal. Methods 2014, 6, 7294-7304. [CrossRef]

20. Sousa, J.C.G.; Ribeiro, A.R.; Barbosa, M.O.; Pereira, M.F.R.; Silva, A.M.T. A review on environmental monitoring of water organic pollutants identified by eu guidelines. J. Hazard. Mater. 2018, 344, 146-162. [CrossRef] [PubMed]

21. Larsson, E.; al-Hamimi, S.; Jönsson, J.Å. Behaviour of nonsteroidal anti-inflammatory drugs and eight of their metabolites during wastewater treatment studied by hollow fibre liquid phase microextraction and liquid chromatography mass spectrometry. Sci. Total Environ. 2014, 485-486, 300-308. [CrossRef] [PubMed]

22. Payán, M.R.; López, M.Á.B.; Torres, R.F.; Navarro, M.V.; Mochón, M.C. Electromembrane extraction (eme) and hplc determination of non-steroidal anti-inflammatory drugs (nsaids) in wastewater samples. Talanta 2011, 85, 394-399. [CrossRef] [PubMed]

23. Mamat, N.A.; See, H.H. Development and evaluation of electromembrane extraction across a hollow polymer inclusion membrane. J. Chromatogr. A 2015, 1406, 34-39. [CrossRef] [PubMed]

24. See, H.H.; Hauser, P.C. Electro-driven extraction of low levels of lipophilic organic anions and cations across plasticized cellulose triacetate membranes: Effect of the membrane composition. J. Membr. Sci. 2014, 450, 147-152. [CrossRef]

25. See, H.H.; Stratz, S.; Hauser, P.C. Electro-driven extraction across a polymer inclusion membrane in a flow-through cell. J. Chromatogr. A 2013, 1300, 79-84. [CrossRef] [PubMed]

26. Padrón, M.E.T.; Ferrera, Z.S.; Rodríguez, J.J.S. Coupling of solid-phase microextraction with micellar desorption and high performance liquid chromatography for the determination of pharmaceutical residues in environmental liquid samples. Biomed. Chromatogr. 2009, 23, 1175-1185. [CrossRef]

27. Ribeiro, A.R.; Pedrosa, M.; Moreira, N.F.F.; Pereira, M.F.R.; Silva, A.M.T. Environmental friendly method for urban wastewater monitoring of micropollutants defined in the directive 2013/39/eu and decision 2015/495/eu. J. Chromatogr. A 2015, 1418, 140-149. [CrossRef] [PubMed]

28. Santos, L.H.M.L.M.; Araújo, A.N.; Fachini, A.; Pena, A.; Delerue-Matos, C.; Montenegro, M.C.B.S.M. Ecotoxicological aspects related to the presence of pharmaceuticals in the aquatic environment. J. Hazard. Mater. 2010, 175, 45-95. [CrossRef] [PubMed]

29. Arous, O.; Kerdjoudj, H.; Seta, P. Comparison of carrier-facilitated silver (i) and copper (ii) ions transport mechanisms in a supported liquid membrane and in a plasticized cellulose triacetate membrane. J. Membr. Sci. 2004, 241, 177-185. [CrossRef]

30. Mao, X.; He, M.; Chen, B.; Hu, B. Membrane protected c18 coated stir bar sorptive extraction combined with high performance liquid chromatography-ultraviolet detection for the determination of non-steroidal anti-inflammatory drugs in water samples. J. Chromatogr. A 2016, 1472, 27-34. [CrossRef] [PubMed] 
31. Li, N.; Chen, J.; Shi, Y.-P. Magnetic polyethyleneimine functionalized reduced graphene oxide as a novel magnetic sorbent for the separation of polar non-steroidal anti-inflammatory drugs in waters. Talanta 2019, 191, 526-534. [CrossRef] [PubMed]

32. Wang, R.; Li, W.; Chen, Z. Solid phase microextraction with poly(deep eutectic solvent) monolithic column online coupled to hplc for determination of non-steroidal anti-inflammatory drugs. Anal. Chim. Acta 2018, 1018, 111-118. [CrossRef] [PubMed]

33. Abd Wahib, S.M.; Wan Ibrahim, W.A.; Sanagi, M.M.; Kamboh, M.A.; Abdul Keyon, A.S. Magnetic sporopollenin-cyanopropyltriethoxysilane-dispersive micro-solid phase extraction coupled with high performance liquid chromatography for the determination of selected non-steroidal anti-inflammatory drugs in water samples. J. Chromatogr. A 2018, 1532, 50-57. [CrossRef] [PubMed]

(C) 2020 by the authors. Licensee MDPI, Basel, Switzerland. This article is an open access article distributed under the terms and conditions of the Creative Commons Attribution (CC BY) license (http://creativecommons.org/licenses/by/4.0/). 